\title{
PADRÕES DE SONO, HÁBITOS E COMPOR- TAMENTOS ALIMENTARES DE CRIANÇAS COM SOBREPESO/OBESIDADE E SUAS CUIDADORAS
}

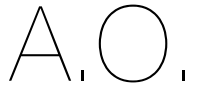

ARTIGO ORIGINAL

1 Centro de Investigação em Psicologia (CIPsi), Escola de Psicologia da Universidade do Minho, Campus de Gualtar, 4710-057 Braga, Portuga

2 Faculdade de Ciências da Nutrição e Alimentação da Universidade do Porto,

Rua do Campo Alegre,

n. ${ }^{\circ} 823$,

4150-180 Porto, Portugal

${ }^{3}$ Centro Materno

Pediátrico do Centro

Hospitalar Universitário de

São João,

Alameda Prof. Hernâni

Monteiro,

4200-319 Porto, Portugal

`Endereço para correspondência:

Sofia Ramalho

Escola de Psicologia da

Universidade do Minho,

Campus de Gualtar,

4710-057 Braga, Portuga

sofia.mm.ramalho@gmail.com

Histórico do artigo

Recebido a 27 de outubro de 2020 Aceite a 8 de abril de 2021

\section{SLEEP PATTERNS, EATING HABITS AND BEHAVIORS OF CHILDREN WITH OVERWEIGHT/OBESITY AND THEIR CAREGIVERS}

Sofia M Ramalho1"; Diana e Silva ${ }^{2,3}$; Cristiana Costa²; Eva Conceição

RESUMO

INTRODUÇÃo: A má higiene do sono pode ter impacto negativo na eficácia do tratamento da obesidade em idade pediátrica. OBJETIVOS: Determinar as associações existentes entre os padrões de sono-vigília, hábitos de ingestão alimentar e comportamentos alimentares problemáticos de crianças em tratamento hospitalar para excesso de peso e os padrões de sono-vigília e comportamentos alimentares das suas cuidadoras.

METODOLOGIA: Foram avaliadas 110 crianças Portuguesas (Faixa etária: 8 - 12 anos;10,13 \pm 1,39 anos; 64 Raparigas; z-score Índice de Massa Corporal 2,72 $\pm 0,65$ ) em tratamento hospitalar para pré-obesidade/obesidade e respetivas cuidadoras ( $\mathrm{n}=110$; 39,84 $\pm 5,48$ anos; Índice de Massa Corporal 29,55 $\pm 5,87 \mathrm{~kg} / \mathrm{m}^{2}$ ). Crianças e cuidadoras reportaram os seus horários de sono nos últimos 7 dias e responderam a um conjunto de questionários de autorrelato (Crianças: Questionário de Frequência Alimentar; Teste de Atitudes Alimentares para Crianças; Cuidadoras: Questionário de Três Fatores do Comportamento Alimentar). Análises estatísticas correlacionais e de diferenças entre grupos foram realizadas.

RESULTADOS: Crianças com obesidade apresentaram uma média de horas de sono significativamente inferior (pré-obesidade: 9h42 \pm Oh54 horas; obesidade: 9h11 \pm Oh49 horas; $p=0,026$ ). A irregularidade nas horas de deitar entre os dias de semana/fim de semana, associou-se a maior frequência de ingestão de "bolachas" $\left(r_{s}=0,24, p=0,015\right)$ e de "bolos" $(r=0,23, p=0,023)$. Horas tardias de acordar/deitar das crianças associaram à menor restrição cognitiva em relação à alimentação por parte das cuidadoras $\left(r_{s}=-0,20, p=0,034 ; r_{s}=-0,23, p=0,017\right)$

CONCLUSÕES: Número insuficiente de horas de sono e padrões de sono irregulares entre dias da semana e fim de semana das crianças associaram-se a hábitos alimentares pouco saudáveis e a alterações do comportamento alimentar nas suas cuidadoras.

\section{PALAVRAS-CHAVE}

Comportamento alimentar, Crianças, Hábitos alimentares, Obesidade, Padrões de sono

\section{ABSTRACT}

INTRODUCTION: Poor sleep hygiene can have a negative impact on the effectiveness of pediatric obesity treatment.

OBJECTIVES: The aim of this study was to explore associations between sleep patterns, food/beverages consumption and maladaptive eating behaviors of children under hospital treatment for overweight/obesity and the sleep patterns and maladaptive eating behaviors of their caregivers.

METHODOLOGY: This cross-sectional study assessed 110 Portuguese children (Age range: 8-12 years; $10.13 \pm 1.39$ years; 64 girls Body Mass Index z-score $2.74 \pm 0.66$ ) in hospital ambulatory treatment for overweight/obesity and their caregivers $(n=110$; age $39.84 \pm 5.48$ years; Body Mass Index $\left.29.55 \pm 5.87 \mathrm{~kg} / \mathrm{m}^{2}\right)$. Children and caregivers reported their sleep patterns in the last 7 days, and answered to a set of self-report measures (Children: Food Frequency Questionnaire; Children's Eating Attitudes Test; Caregivers: Three-Factor Eating Questionnaire). Differences between groups were tested and correlational analyzes performed. RESULTS: Children with obesity presented a lower average of sleep hours (pre-obesity: 9:42 \pm 0h54 hours; obesity: 9h11 \pm 0h49 hours; $p=0.026)$. Bedtime irregularity between weekdays/weekends was associated with a higher frequency of "cookies" $\left(r_{s}=0.24\right.$, $\mathrm{p}=0.015)$ and "pastries" $(r=0.23, \mathrm{p}=0.023)$ intake. Children's late-waking/sleeping times were associated with reduced cognitive restriction of caregivers concerning food $\left(r_{s}=-0.20, p=0.034 ; r_{s}=-0.23, p=0.017\right)$. CONCLUSIONS: Children's insufficient number of hours of sleep and irregular sleep patterns between weekdays and weekends were associated with unhealthy eating habits and caregivers' problematic eating behaviors.

KEYWORDS

Eating behavior, Children, Dietary patterns, Obesity, Sleep patterns 


\section{INTRODUÇÃO}

Vários estudos demonstram que uma má higiene do sono na infância, caracterizada por padrões de sono de curta duração e irregularidade dos padrões de sono-vigília está associada a um aumento ponderal excessivo ao longo do tempo $(1,2)$.

De facto no que concerne à qualidade da alimentação, padrões de sono de curta duração, em conjunto com horas de deitar tardias e padrões de sono irregulares parecem estar correlacionados com a ingestão de alimentos energeticamente densos (e.g produtos com açúcares adicionados, sumos e refrigerantes, chocolates, snacks e Fast-food) e a uma menor ingestão de alimentos nutricionalmente densos (e.g. fruta, produtos hortícolas e peixe) (3).

Por outro lado, a investigação também indica que padrões de sono de curta duração dos pais podem influenciar negativamente o comportamento das crianças/adolescentes em relação aos seus próprios hábitos de sono (hora de deitar/acordar), quer pelo facto de estas dormirem com os pais, quer por fatores socioeconómicos (4). Efetivamente, a duração e a qualidade dos padrões de sono dos pais constituem preditores significativos dos padrões de sono de crianças/ adolescentes, sendo estas associações mais fortes entre a criança/ adolescente e a sua mãe/cuidadora (4). Contudo, a literatura tem se focado maioritariamente nos padrões de sono-vigília das crianças/ adolescentes. Por essa razão são necessários mais estudos integrativos que considerem simultaneamente comportamento alimentar e padrões de sono-vigília maternos, já que a má higiene do sono da criança/ adolescente pode ter impacto negativo na eficácia do tratamento da obesidade em idade pediátrica.

\section{OBJETIVOS}

O presente estudo tem como principal objetivo investigar de que forma os padrões de sono-vigília, hábitos e comportamentos alimentares problemáticos de crianças em tratamento hospitalar para excesso de peso se relacionam com os padrões de sono-vigília e comportamentos alimentares das suas cuidadoras. Objetivos específicos: 1) Descrever e examinar diferenças entre crianças com pré-obesidade e obesidade ao nível da duração e padrões de sono; e 2) Avaliar a existência de associações entre parâmetros antropométricos (Índice de Massa Corporal (IMC), z-score de IMC, percentagem de massa gorda, perímetro da cintura) das crianças e das suas cuidadoras; 3) Examinar a existência de associações entre o número médio de horas de sono por noite e a irregularidade dos padrões de sono-vigília com a frequência de consumo alimentar e comportamentos alimentares problemáticos das crianças e das suas cuidadoras.

\section{METODOLOGIA}

\section{Desenho do Estudo/Procedimentos}

Trata-se de um estudo transversal com uma amostra de conveniência. A recolha de dados decorreu entre Junho de 2017 e Março de 2018. O estudo foi aprovado pelas comissões de ética do Centro Hospitalar Universitário de São João (CHUSJ) e da Universidade do Minho Subcomissão de Ética para a Investigação em Ciências da Vida e da Saúde. O consentimento informado foi assinado pelo representante legal que acompanhava o participante na consulta e pelo participante. Todos os participantes que cumpriam os critérios de inclusão foram convidados, de forma sistemática, a integrar o estudo durante o período de recolha de dados no contexto da consulta externa da Unidade de Nutrição Pediátrica do Hospital Pediátrico Integrado - Centro Hospitalar Universitário de São João (CHUSJ). O convite para participação foi realizado em primeiras consultas e em consultas de seguimento.

Foram considerados os seguintes critérios de inclusão: (1) idade compreendida entre os 8 e 12 anos; (2) percentil de IMC $\geq 85$; e (3) ser acompanhado(a) por cuidadora legal ou progenitora disposta a participar no estudo. Critérios de exclusão: (1) presença de obesidade secundária a outras patologias; e (2) existência de perturbação da aprendizagem específica que impedisse a criança e/ou a cuidadora legal/progenitora de ler e compreender texto escrito.

\section{Instrumentos}

Avaliação Antropométrica: A determinação da estatura $(\mathrm{em} \mathrm{cm})$, quer da criança quer da sua cuidadora foi realizada através de estadiómetro Seca ${ }^{\circledR}$ (sensibilidade de $0,1 \mathrm{~cm}$ ). O peso da cuidadora foi avaliado pela balança de marca Seca ${ }^{\circledR}$ modelo 220 (sensibilidade de $0,1 \mathrm{~kg}$ ). A caracterização do estado nutricional das cuidadoras foi realizada através do IMC de Quetelet (em $\left.\mathrm{kg} / \mathrm{m}^{2}\right)$, e de acordo com os pontos de corte definidos pela Organização Mundial da Saúde (OMS). Enquanto que para as crianças o IMC $\left(\mathrm{kg} / \mathrm{m}^{2}\right)$ e a Massa Gorda em percentagem (MG \%) foram determinados através da balança de Bioimpedância TANITA ${ }^{\circledR}$ modelo TBF-300 (com sensibilidade de 0,1 kg). O valor de IMC foi expresso em z-score, calculado através do programa WHO AnthroPlus ${ }^{\circledR}$ e categorizado de acordo com os critérios da OMS. A medição do perímetro da cintura $(\mathrm{em} \mathrm{cm}$ ) da criança considerou o método de Cameron (5). A presença de obesidade abdominal nas crianças foi avaliada através da tabela de referência para a população pediátrica europeia-americana, em função da idade e sexo, de Fernandez e colegas (6). Considerou-se o percentil 75 como marcador de aumento do risco de obesidade abdominal e o percentil 90 como limite acima do qual se define obesidade abdominal (5).

Padrões de Sono-Vigília: A duração de sono e os padrões de sono das crianças e das suas cuidadoras/mães foram estimados através do recordatório em papel dos horários de sono (hora de acordar, deitar e adormecer) para cada dia dos últimos 7 dias. Este mesmo recordatório foi preenchido separadamente por crianças e pelas suas cuidadoras/ mães, de forma individual, com apoio do inquiridor no dia da consulta. As respostas das crianças foram posteriormente confirmadas pelas suas cuidadoras/mães. O recordatório permitiu calcular a média aritmética do número de horas de sono por noite, a irregularidade dos horários de sono-vigília ["número médio de horas de sono por noite nos dias de fim de semana (noites de sexta-feira e sábado)" - "o número médio de horas de sono por noite nos dias de semana"], tal como a irregularidade nas horas de acordar e deitar (diferença absoluta entre a hora média de acordar/deitar nos dias de fim de semana e a hora média de acordar/ deitar nos dias de semana). Valores positivos nas variáveis referentes à irregularidade do sono indicam uma média superior nos dias de fim de semana. Valores negativos demonstram uma média inferior nos dias de semana em comparação com os dias de fim de semana.

\section{Consumo Alimentar/Comportamentos Alimentares}

Questionário de Frequência Alimentar: Para avaliar a frequência de consumo alimentar de hortofrutícolas, açúcar, doces e pastéis, gelados, Fast-food, sumos de fruta e refrigerantes das crianças foi elaborado um questionário de frequência alimentar (QFA). Este questionário teve por base a adaptação de dois questionários de frequência alimentar validados para as crianças portugueses, nomeadamente o questionário do estudo Pro-children (7), para avaliar a ingestão de hortofrutícolas (na última semana) e o Questionário de Frequência Alimentar do Serviço de Higiene e Epidemiologia da Faculdade de Medicina da Universidade do Porto (8) para avaliar a ingestão dos restantes produtos alimentares (no último mês).

Teste de Atitudes Alimentares para Crianças (Children's Eating 
Attitudes Test - ChEAT) (9): Este é um questionário de auto-relato de 26 itens, que se dividem em 4 subescalas: 1) "Medo de Engordar", 2) "Comportamentos Restritivos e Purgativos", 3) "Preocupação com a Comida" e 4) "Pressão Social para Comer". Valores mais elevados correspondem a maior perturbação do comportamento alimentar. Questionário de Três Fatores do Comportamento Alimentar (ThreeFactor Eating Questionnaire - TFEQ-R21) (10): É composto por 21 itens e três subescalas: desinibição alimentar, restrição cognitiva e alimentação emocional. Valores mais elevados indicam maior perturbação do comportamento alimentar em adultos.

\section{Análise Estatística}

A análise descritiva dos dados foi efetuada através do cálculo das médias e desvios padrões. Teste T para Amostras Independentes e de Análise de Variância (ANOVA) unifactorial com Post-Hoc de Gabriel foram realizados para testar diferenças inter-sujeitos. Os equivalentes não paramétricos, teste de Kruskall-Wallis e de Mann-Whitney com correção Bonferroni foram efetuados quando as variáveis não apresentavam uma distribuição normal. Coeficiente de Correlação de Pearson e de Spearman foram realizados para analisar as associações entre as variáveis em estudo. Os dados foram analisados com recurso ao Statistical Package for Social Science - SPSS, versão 22. Valores de p inferiores a 0,05 foram considerados estatisticamente significativos.

\section{RESULTADOS}

\section{Caracterização da Amostra e dos Padrões de Sono-Vigília}

Foram avaliadas 110 crianças, 64 (58,2\%) do sexo feminino, com idades compreendidas entre os 8 e os 12 anos (Média=10,13 $\pm 1,39$ anos) que se encontravam em tratamento para excesso de peso/obesidade em regime ambulatório em média à 23,75 $\$ 32.04$ meses ( mín = 0; máx =161 meses). A Tabela 1 descreve os parâmetros antropométricos, $z$-score de IMC, média de percentagem de massa gorda e de perímetro cintura, em função do sexo e grupo etário das crianças. Cerca de $86,4 \%$ das crianças desta amostra tinha obesidade ( $\geq$ P95) e 93,1\% apresentava obesidade abdominal ( $\geq$ P90 perímetro da cintura).

Verificámos que as crianças com obesidade diferem significativamente das crianças com pré-obesidade ao nível do número médio de horas de sono, apresentando uma média de horas de sono por noite inferior (pré-obesidade: 9h42 \pm Oh54; obesidade: 9h11 $\pm 0 h 49$; t (108) = 2,23, $p=0,026$ ).

A idade média das cuidadoras $(n=110)$ é de 39,84 $\pm 5,48$ anos (mín. = 24 anos e máx. $=52$ anos), a maioria reportou ter completo o $3 .{ }^{\circ}$ ciclo do ensino básico ( $n=39 ; 35,5 \%)$, ser casada $(n=75 ; 68,2 \%)$ e estar empregada a tempo inteiro $(n=77 ; 70,0 \%)$. Da caracterização do estado nutricional, observa-se que a média de IMC das cuidadoras é 29,55 $\pm 5,87$ $\mathrm{kg} / \mathrm{m}^{2}$ (mín. = 18,6 kg/m² e máx. $=46,3 \mathrm{~kg} / \mathrm{m}^{2}$ ) e que $81,5 \%$ destas apresentava pré-obesidade ou obesidade de acordo com os critérios da OMS.

\section{Associação entre Padrões de Sono-Vigília, Estado Nutricional e Hábitos/Comportamentos Alimentares Problemáticos das Crianças e suas Cuidadoras}

O número médio de horas de sono por semana das crianças correlacionou-se positivamente com a frequência de consumo de "sopa de legumes" $\left(r_{s}=0,206, p=0,039\right)$. Observámos ainda uma correlação positiva entre a hora média de deitar com a frequência de consumo de "pizza" $\left(r_{s}=0,212, p=0,035\right)$ e que uma maior irregularidade da hora média de deitar entre o fim de semana e dias de semana correlacionava-se positivamente com a frequência de consumo de "bolachas ou biscoitos" ( $\left.r_{s}=0,244, p=0,015\right)$ e de "croissants, pastéis ou bolos caseiros" ( $\left.r_{s}=0,229, p=0,023\right)$.A Tabela 2 descreve as associações entre os padrões de sono-vigília, estado nutricional e comportamentos alimentares problemáticos das crianças e suas cuidadoras. Uma média superior de horas de sono por noite das crianças correlacionou-se com um menor $z$-score de IMC $(r=-0,22, p=0,020)$ e com menor perímetro da cintura $(r=-0,30, p=0,002)$. Tal como uma maior irregularidade dos horários de sono-vigília das crianças, caracterizada por um menor número de horas de sono nos dias de fim de semana em comparação com os dias de semana, se associou a um z-score de IMC superior $\left(r_{s}=-0,20, p=0,032\right)$. No que concerne a comportamentos alimentares problemáticos, uma média superior da hora e acordar e de deitar das crianças demonstrou estar associada à menor restrição cognitiva por parte das cuidadoras $\left(r_{s}=-0,20, p=0,034 ; r_{s}=-0,23, p=0,017\right)$.

\section{DISCUSSÃO DOS RESULTADOS}

A maioria das crianças em tratamento para excesso de peso em contexto hospitalar que participaram neste estudo cumpriam as recomendações da Sociedade Portuguesa de Pediatria e da National Sleep Foundation em relação ao número de horas de sono diárias (9 a 11h), reportando dormir em média 9h15 minutos por noite. Contudo, observámos que as crianças com obesidade apresentavam uma média de horas de sono por noite inferior às crianças com pré-obesidade. O estudo de Olds e colegas (2011) (11) ilustra o possível efeito da privação de sono no z-score de IMC e nos comportamentos sedentários, observando-se que uma hora de deitar tardia aumenta em 1,5 vezes a probabilidade de uma criança/adolescente apresentar valores superiores de $z$-score de IMC, tendo 1,8 vezes mais probabilidade de ser inativo fisicamente e 3 vezes mais de permanecer longos períodos de tempo à frente de um ecrã.

\section{Tabela 1}

Parâmetros Antropométricos em Função do Sexo e Grupo Etário das Crianças

\begin{tabular}{|c|c|c|c|c|c|c|c|c|}
\hline & & \multirow{2}{*}{$\begin{array}{l}\text { TOTAL } \\
(\mathrm{N}=110)\end{array}$} & \multicolumn{2}{|c|}{ SEXO } & \multicolumn{4}{|c|}{ GRUPO ETÁRIO } \\
\hline & & & $\begin{array}{l}\text { FEMININO } \\
(\mathrm{N}=64)\end{array}$ & $\begin{array}{l}\text { MASCULINO } \\
(\mathrm{N}=46)\end{array}$ & $\mathbf{P}$ & $\begin{array}{c}\geq 8 \mathrm{E}<10 \text { ANOS } \\
(\mathrm{N}=40)\end{array}$ & $\begin{array}{l}\geq 10 \text { ANOS } \\
(N=70)\end{array}$ & P \\
\hline \multirow{2}{*}{ z-score de IMC } & Média (DP) & $2,72(0,65)$ & $2,66(0,63)$ & $2,80(0,67)$ & \multirow{2}{*}{0,272} & $3,02(0,68)$ & $2,55(0,57)$ & \multirow{2}{*}{$<0,001^{\star \star \star}$} \\
\hline & Mín. - Máx. & $1,34-4,86$ & $1,34-4,86$ & $1,42-4,63$ & & $1,75-4,86$ & $1,34-4,63$ & \\
\hline \multirow{2}{*}{$\begin{array}{l}\text { Massa Gorda } \\
(\%)\end{array}$} & Média (DP) & $34,71(6,08)$ & $35,78(5,80)$ & $33,22(6,21)$ & \multirow{2}{*}{$0,047^{\star}$} & $35,32(6,21)$ & $34,42(6,04)$ & \multirow{2}{*}{0,507} \\
\hline & Mín. - Máx. & $18,7-48,0$ & $18,7-48,0$ & $20,7-46,6$ & & $18,7-48,0$ & $20,7-46,6$ & \\
\hline \multirow{2}{*}{$\begin{array}{l}\text { Perímetro da cintura } \\
\text { (cm) }\end{array}$} & Média (DP) & $89,10(9,70)$ & $88,06(9,45)$ & $90,48(9,96)$ & \multirow{2}{*}{0,212} & $85,21(9,73)$ & $91,22(9,06)$ & \multirow{2}{*}{$0,002^{\star \star}$} \\
\hline & Mín. - Máx. & $62,9-113,2$ & $69,5-113,0$ & $62,9-113,2$ & & $62,9-113,0$ & $74,5-113,2$ & \\
\hline
\end{tabular}

${ }^{*} p<0,05$

${ }^{\star \star} p<0,01$

${ }^{\star \star \star} \mathrm{p}<0,001$
DP: Desvio-Padrão

IMC: Índice de Massa Corporal

Teste T para amostras independentes 


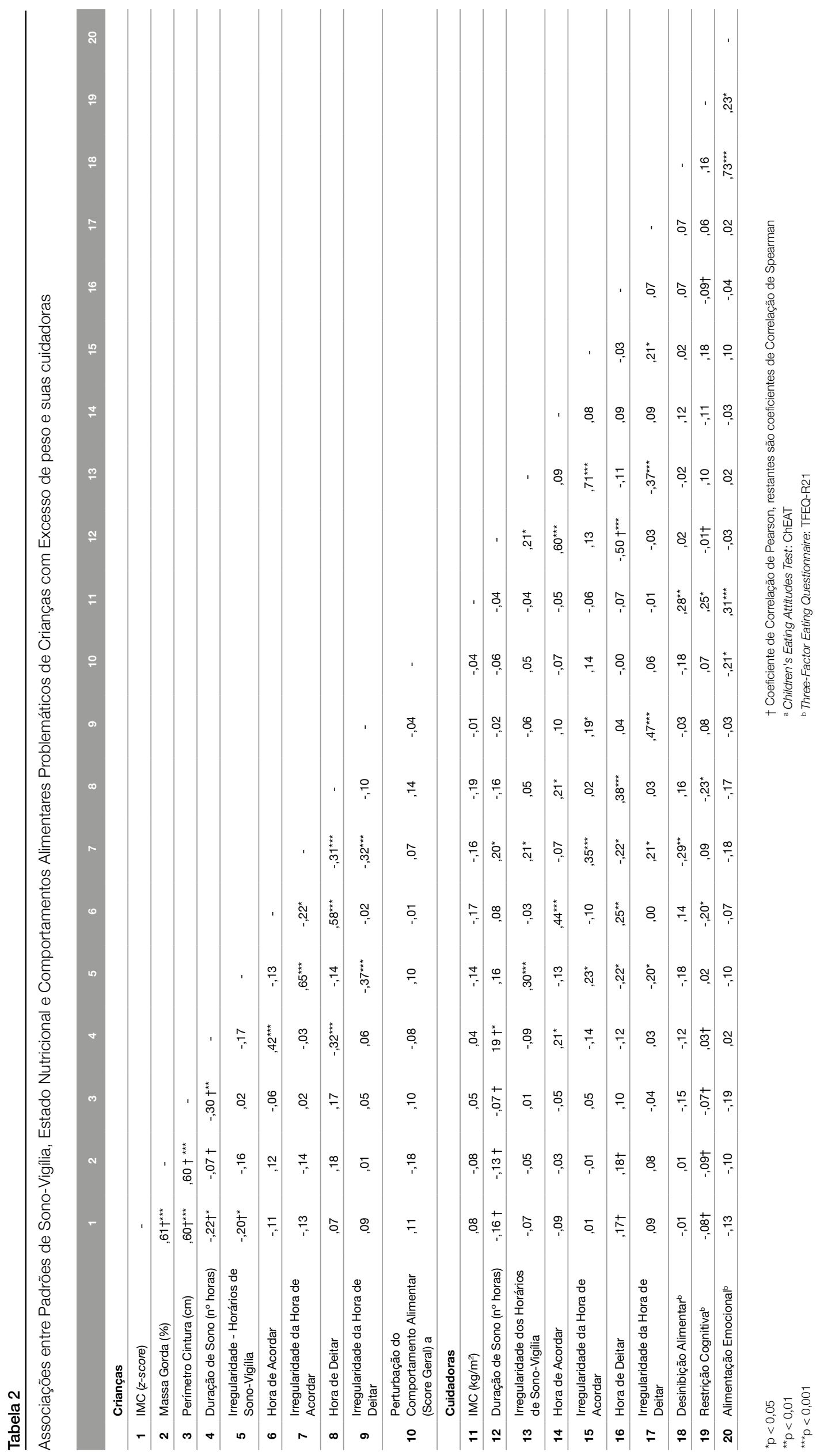


Paralelamente, a evidência científica suporta a associação entre padrões de sono-vigília e a exposição a um ambiente obesogénico em idade pediátrica, sendo este caracterizado pelo excessivo consumo de alimentos nutricionalmente desiquilibrados (3). O presente estudo revelou que crianças que se deitam mais tarde reportam um maior consumo de snacks poucos saudáveis. Tal parece estar associado não só à hora de deitar, mas também à irregularidade nas horas de deitar entre os dias de semana e de fim de semana, uma vez que na nossa amostra este indicador demonstrou estar associado a uma maior frequência de ingestão de "pastéis/bolos caseiros", "bolachas/ biscoitos e croissants" e "pizza". No que concerne à frequência do consumo de hortofrutícolas, a privação de sono associou-se a uma menor frequência de consumo de "sopa de legumes" nas crianças. Estes resultados vão de encontro à literatura que indica que $1 \mathrm{~h}$ adicional na duração média de sono leva a um aumento significativo no consumo de fruta e vegetais e a uma diminuição do consumo de alimentos ricos em açúcar e gorduras (12). Estes dados reforçam a importância de intervir nos padrões de sono-vigília no contexto da obesidade em idade pediátrica pelo impacto positivo que esta intervenção pode ter ao nível da regulação do sistema neuroendócrino, do metabolismo e em última instância da ingestão alimentar.

De forma geral, os resultados deste estudo são suportados pelo tamanho da amostra, pela qualidade da informação recolhida (recordatório do sono realizado de forma individual com confirmação posterior das respostas das crianças pelas mães/cuidadoras), e pela inclusão de múltiplas medidas antropométricas e de composição corporal. Contudo, na interpretação dos resultados deste estudo devem ser tidas em conta algumas limitações, tais como o desenho transversal que impossibilita conclusões de causa/efeito, não ter sido considerado o impacto de possíveis fatores confundidores nas correlações testadas (e.g. características sociodemográficas), e a limitação geográfica da recolha de dados (região norte de Portugal). Não obstante, a escassez de estudos sobre esta temática a nível nacional, especificamente com população pediátrica acompanhada em ambiente hospitalar para perda de peso, justifica a sua pertinência refletindo a importância de não só serem considerados estudos futuros na população com obesidade como a inclusão de grupos de controlo e de variáveis parentais.

\section{CONCLUSÕES}

Um número insuficiente de horas de sono e padrões de sono irregulares entre dias da semana e fim de semana associaram-se a hábitos alimentares pouco saudáveis e a alterações do comportamento alimentar nas crianças e suas mães/cuidadoras. Mães/cuidadoras e a irregularidade dos seus padrões de sono-vigília parecem ter um papel determinante nos hábitos de sono das crianças em tratamento para excesso de peso. Assim profissionais de saúde em contacto com a problemática da obesidade pediátrica devem estar sensíveis à importância de melhorar a higiene do sono das crianças, tal como das suas mães/cuidadoras.

\section{FINANCIAMENTO}

Este estudo foi parcialmente realizado no Centro de Investigação em Psicologia (CIPsi/UM) da Escola de Psicologia da Universidade do Minho, apoiado pela Fundação para a Ciência e a Tecnologia (FCT) através do Orçamento do Estado Português (UIDB/01662/2020) e financiado (parcialmente) através de fundos concedidos a Eva Conceição (2020.01538.CEECIND; POCI-01-0145-FEDER-028209). A Fundação para a Ciência e Tecnologia não teve qualquer papel no design, recolha, análise e interpretação dos dados, assim como na redação do manuscrito, ou na decisão de enviar o manuscrito para publicação.

\section{REFERÊNCIAS BIBLIOGRÁFICAS}

1. Wu Y, Gong Q, Zou Z, Li H, Zhang X. Short sleep duration and obesity among children: A systematic review and meta-analysis of prospective studies. Obes Res Clin Pract. 2017;11(2):140-50.

2. Burt J, Dube L, Thibault L, Gruber R. Sleep and eating in childhood: A potential behavioral mechanism underlying the relationship between poor sleep and obesity. Sleep Med. 2014;15(1):71-5.

3. Tambalis KD, Panagiotakos DB, Psarra G, Sidossis LS. Insufficient Sleep Duration Is Associated With Dietary Habits, Screen Time, and Obesity in Children. J Clin Sleep Med. 2018;14(10):1689-96.

4. Zhang J, Li AM, Fok TF, Wing YK. Roles of Parental Sleep/Wake Patterns, Socioeconomic Status, and Daytime Activities in the Sleep/Wake Patterns of Children. J Pediatr. 2010;156(4).

5. Rito A, Breda J, do Carmo I, Instituto Nacional de Saúde Doutor Ricardo Jorge, Direção-Geral da Saúde. Guia de Avaliação do Estado Nutricional Infantil e Juvenil. Instituto Nacional de Saúde Doutor Ricardo Jorge (INSA, IP). Lisboa; 2011.

6. Fernández JR, Redden DT, Pietrobelli A, Allison DB. Waist circumference percentiles in nationally representative samples of African-American, European-American, and Mexican-American children and adolescents. J Pediatr. 2004;145(4):439-44.

7. Yngve A, Wolf A, Poortvliet E, Elmadfa I, Brug J, Ehrenblad B, et al. Fruit and vegetable intake in a sample of 11-year-old children in 9 European countries: The pro children cross-sectional survey. Annals of Nutrition and Metabolism. 2005;49:236-45.

8. Departamento de Higiene e Epidemiologia. Questionário de frequência alimentar (QFA). Available from: http://higiene.med.up.pt/freq.php.

9. Teixeira M, Pereira A, Saraiva J, Marques M, Soares M, Bos Carvalho S, et al. Portuguese validation of the Children's Eating Attitudes Test. Rev Psiq Clín. 2012;39(6):189-93. 10. Duarte PAS, Palmeira L, Pinto-Gouveia J. The Three-Factor Eating Questionnaire-R21: a confirmatory factor analysis in a Portuguese sample. Eat Weight Disord - Stud Anorexia, Bulim Obes. 2020;25:247-256.

11. Olds TS, Maher CA, Matricciani L. Sleep duration or bedtime? Exploring the relationship between sleep habits and weight status and activity patterns. Sleep. 2011;34(10):1299-307.

12. Pérez-Farinós N, Villar-Villalba C, López Sobaler AM, Dal Re Saavedra MÁ, Aparicio A, Santos Sanz S, et al. The relationship between hours of sleep, screen time and frequency of food and drink consumption in Spain in the 2011 and 2013 ALADINO: a cross-sectional study. BMC Public Health. 2017 Jan 6;17(33):1-12. 